DOI: $10.1002 /(($ please add manuscript number $))$

Article type: Communication

\title{
Semiconducting Nanowire-based Optoelectronic Fibers
}

Wei Yan, Yunpeng Qu, Tapajyoti Das Gupta, Arouna Darga, Dang Tùng Nguyên, Alexis Gérald Page, Mariana Rossi, Michele Ceriotti, and Fabien Sorin*

W. Yan, Dr. Y. Qu, Dr. T. D. Gupta, D. T. Nguyên, A. G. Page, Prof. F. Sorin

Laboratory of Photonic Materials and Fibre Devices (FIMAP), Institute of Materials, Ecole

Polytechnique Fédérale de Lausanne (EPFL)

Lausanne 1015, Switzerland

* E-mail: fabien.sorin@epfl.ch

Dr. M. Rossi, Prof. M. Ceriotti

Laboratory of Computational Science and Modeling (COSMO), Institute of Materials, Ecole

Polytechnique Fédérale de Lausanne (EPFL)

Lausanne 1015, Switzerland

Dr. A. Darga

Group of Electrical Engineering of Paris (GeePs)

91192 Gif sur Yvette Cedex, France

Sorbonne Iniversités, UPMC Univ Paris 06

UMR 8507, Paris, France

Keywords: (functional fibers, semiconducting nanowires, optoelectronic devices, semiconductor fibers, multi-material fibers) 


\begin{abstract}
:
The recent ability to integrate semiconductor-based optoelectronic functionalities within thin fibers is opening intriguing opportunities for flexible electronics and advanced textiles. The scalable integration of high quality semiconducting devices within functional fibers remains however a challenge. It is difficult with current strategies to combine high light absorption, good microstructure and efficient electrical contact. The growth of semiconducting nanowires is a great tool to control crystal orientation and ensure a combination of light absorption and charge extraction for efficient photodetection. Thus far however, leveraging the attributes of nanowires has remained seemingly incompatible with fiber materials, geometry, and processing approaches. Here we demonstrate for the first time the integration of semiconducting nanowire based devices at the tip and along the length of polymer fibers. We combine the scalable thermal drawing process with a simple sonochemical treatment to grow nanowire out of electrically addressed amorphous selenium domains. First principles density-functional theory calculations show that this approach enables to tailor the surface energy of crystal facets and favors nanowire growth along a preferred orientation, resulting in fiber-integrated devices of unprecedented performance. We exploit this novel platform to demonstrate an all-fiber-integrated fluorescence imaging system, highlighting novel opportunities in sensing, advanced optical probes, and smart textiles.
\end{abstract}


The recent ability to position electrically conducting and semiconducting domains at prescribed positions within optical fibers is opening a breadth of novel opportunities for advanced medical and biological probes, ${ }^{[1,2]}$ flexible smart sensors and imaging systems, ${ }^{[3-7]}$ nanophotonics, ${ }^{[8]}$ light generation and communication, ${ }^{[1]}$ energy harvesting ${ }^{[9-11]}$ and advanced textiles. ${ }^{[12,13]}$ To functionalize optical fibers, a first strategy relies on wafer-based techniques ${ }^{[8]}$ and modified deposition ${ }^{[14,15]}$ to integrate functional materials at the tip or within a few tens of centimeters of microstructured silica fibers. An alternative approach exploits the well-established thermal drawing of macroscopic preforms that integrate the desired multi-material architecture. ${ }^{[1]}$ This has the advantage of simplicity and scalability, since the fiber pulling step results in tens of kilometers of fibers that have the same cross-sectional structure as the initial preform. Thus far however, realizing high quality semiconducting materials that can act as highperformance photodetectors using the thermal drawing process remains a challenge. A recent strategy consists in the thermal drawing within silica fibers of high melting point materials such as silicon, ${ }^{[16]}$ germanium ${ }^{[17]}$ or various compounds. ${ }^{[18]}$ The melting and solidification during drawing result however in semiconductors with a highly polycrystalline microstructure, requiring local post-drawing annealing or laser-based steps to engineer a desired microstructure. ${ }^{[15,18,19]}$ It is also difficult to integrate electrodes in contact with the semiconducting domains and despite an ingenious and promising method, no device with good optoelectronic properties has been shown. ${ }^{[17]}$ The alternative strategy relies on exploiting the polymer fiber platform. It has several advantages compared to its silica counterpart, including low temperature processing, robust mechanical properties, simple integration of electrodes, and the ability to impart fibers with complex architectures and multiple functionalities. ${ }^{[1]}$ Thus far however, the postdrawing crystallization schemes applied to semiconducting chalcogenide glasses have resulted in poor control over the phase, grain size and orientation, impairing device performance. ${ }^{[5,20]}$

A powerful approach to control the microstructure and enhance optoelectronic performance is via the growth of well-oriented semiconducting nanowires. This approach has not been exploited in the frame of fiber-integrated devices since conventional fabrication procedures of semiconducting nanowires are 
complex and only adapted to specific substrates seemingly incompatible with the thermal drawing process and the fiber material and geometry. ${ }^{[8,21]}$ Here, we demonstrate for the first time the robust and scalable integration of high quality single crystal semiconducting nanowire-based optoelectronic devices at the tip and along the length of polymer optical fibers. We combine the thermal drawing process with a simple sonochemical nanowire growth mechanism applied to amorphous selenium (Se) domains. Selenium is an excellent optoelectronic material in its trigonal phase and is used in a myriad of applications. ${ }^{[22]}$ As we show experimentally and via first principles density-functional theory calculations, its trigonal structure exhibits an exacerbated anisotropy in the surface energy of the different crystal planes in the solvent. This allows us to control the phase and orientation of crystalline nanowires that grow along the desired axis in a simple way, directly in intimate contact with built-in electrodes. The resulting nanowire-based photodetecting devices exhibit an unprecedented combination of excellent optical and optoelectronic properties in terms of light absorption, responsivity, sensitivity and response speed. Moreover, thanks to the polymer fiber platform, the nanowires can be positioned anywhere on the fiber cross-section allowing for the integration of complex and unprecedented functionalities. We demonstrate in particular an architecture with two nanowire-based devices positioned around a stepindex optical fiber, allowing to perform fluorescence imaging using a single multi-functional fiber. Finally, our approach also enables the fabrication of nanowire-based devices along an extended length of flexible fibers, without requiring high-resolution contacting techniques, paving the way towards novel opportunities in flexible optoelectronics and advanced textiles.

We start by investigating the fiber-integrated nanowire mesh formation. A first multi-material fiber (later referred to as the "precursor fiber") was fabricated via the thermal drawing of a macroscopic preform, as schematically illustrated in Figure 1a. The preform consisted of a rectangular Se core placed in intimate contact with carbon-loaded polycarbonate (CPC) nanocomposite domains that act as 

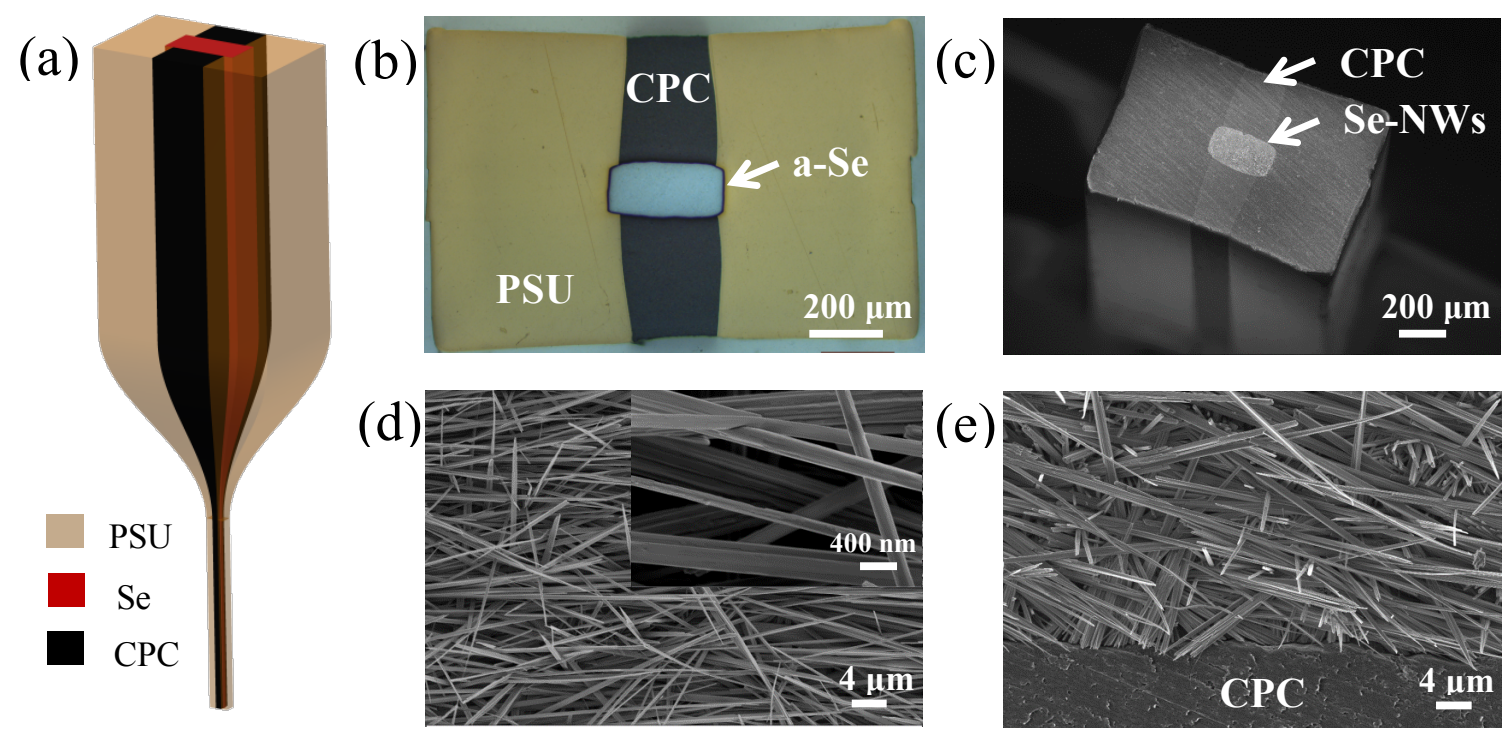

Figure 1. a) Schematic of multi-material thermal drawing. b) Optical photograph of the cross-section of the as-drawn fiber. c) SEM micrograph of the fiber cross-section after Se-NW formation. d) High magnification SEM micrograph with an inset zoom in on individual nanowires. e) SEM micrograph of nanowires contacting $\mathrm{CPC}$.

electrical contacts and run along the entire fiber length. This assembly was encapsulated within a polysulfone (PSU) cladding, a thermoplastic that ensures the mechanical integrity of the resulting fiber (see details in Supplemental Information (SI) 1). The composite structure was subsequently drawn at a temperature above the glass transition of PSU and CPC and the melting point of Se (Figure S1c, SI), into a hundred-meter-long fiber having a cross-section exhibiting an exact scaled down version of the initial preform (Figure 1b and 1c). X-ray diffraction as well as selected-area electron diffraction (SAED) from transmission electron microscope (TEM) characterization show that Se is amorphous after thermal drawing (Figure S1d, SI). To grow Se nanowires on the fiber cross-section, the tip was first polished to ensure a good interface between a-Se and the CPC electrodes. It was then treated in an ultrasonic bath for 1 minute to induce a cavitation effect that is believed to accelerate nucleation in the amorphous Se matrix. ${ }^{[23]}$ Subsequently, the fiber tip was immersed in 1-propanol (a solvent that can dissolve amorphous $\mathrm{Se}^{[24]}$ ) for varying times (a few hours to a few days) at ambient conditions. The Scanning electron 
microscopy (SEM) image of a fiber tip immersed for five days shown in Figure 1d reveals that a high density array of Se nanowires was synthesized between the CPC electrodes (Figure 1d). The diameter of the nanowires is around $200 \mathrm{~nm}$, as shown in the magnified SEM image (Figure 1d), and the nanowire mesh intimately interfaces with the CPC electrodes (Figure 1e).

Se nanowires have been previously fabricated from amorphous nanoparticle seeds obtained by the reduction of Se-based compounds via a hydrothermal approach. ${ }^{[22,25]}$ Here we found that Se nanowires can be synthesized directly from amorphous bulk at room temperature. The SEM micrograph of Figure 2a reveals that nanowires grow from the nuclei formed in the amorphous matrix. From TEM analysis, as shown in Figure $2 b$, the SAED pattern of a typical nanowire obtained by focusing the electron beam along the [100] zone axis indicates that the nanowires preferentially grow along the [001] direction. This pattern can be indexed as the trigonal phase. The lattice constants calculated from the pattern $(\mathrm{a}=0.432$ $\mathrm{nm}, \mathrm{c}=0.493 \mathrm{~nm})$ correspond well to the ones of the Se trigonal phase reported in literature $(\mathrm{a}=0.436$ $\mathrm{nm}, \mathrm{c}=0.495 \mathrm{~nm}) .{ }^{[26]}$ Several diffraction patterns taken from different regions of an individual nanowire were essentially the same, revealing nanowires having a rather monocrystalline nature with little misorientation or grain boundaries. A high-resolution TEM image (HRTEM) taken from the nanowire shows a fringe spacing $(\sim 0.5 \mathrm{~nm})$ that agrees well with the separation between the (001) lattice planes, confirming a growth along the [001] direction (Figure 2c).

To further gain insight into the formation mechanism of these nanowires and their preferred crystallographic orientation, we performed first principles density-functional theory calculations using the PBE exchange correlation functional and many-body van der Waals scheme (MBD) ${ }^{[27,28]}$ using the FHI-aims code. ${ }^{[29]}$ The bulk structural properties computed in this way agree well with experimental data (Table S1, SI2). To test whether the anisotropy of the surface energy can provide a thermodynamic drive for the growth of nanowires, we calculated the surface energies for a few high-symmetry directions of the Se crystal (space group P3121), namely the (010) and (01-1) planes (Figure 2d), as well as the basal plane (001) surface. The surface energies we thus obtained are $\gamma(010)=0.009, \gamma(01-1)$ 
(a)

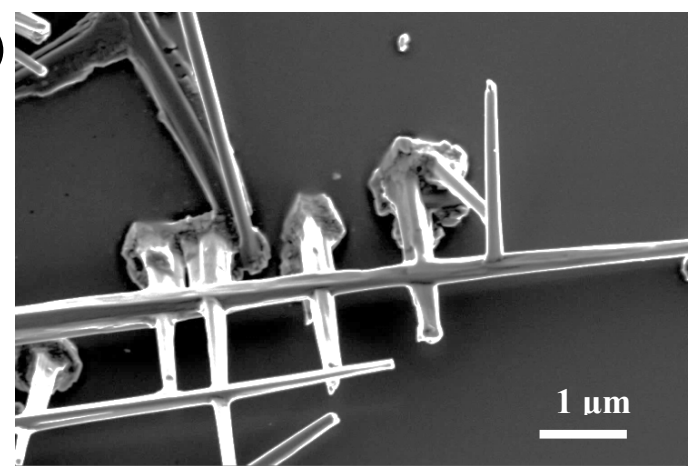

(c)

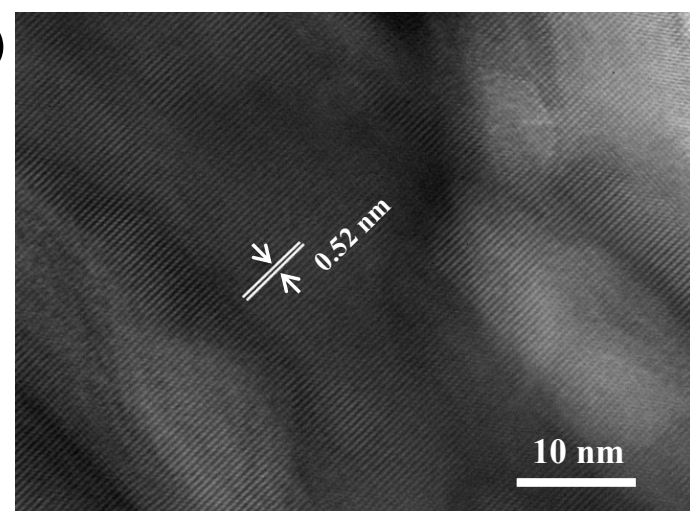

(b)

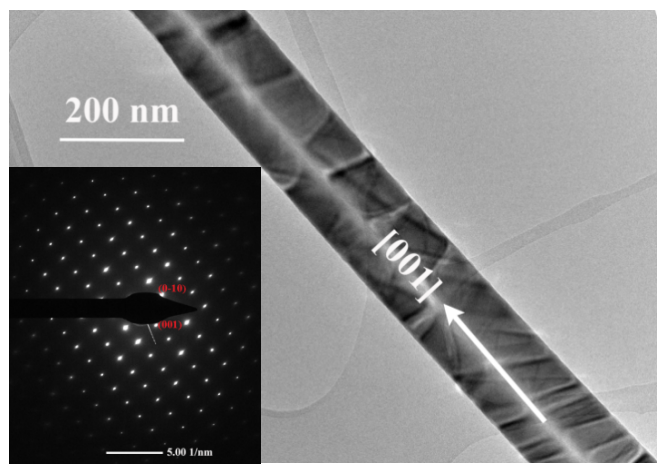

(d)

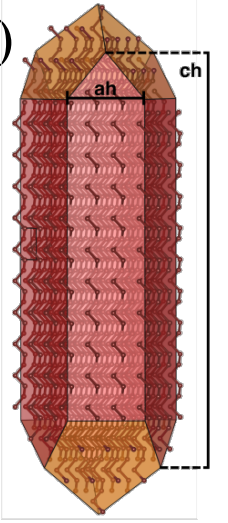

(e)

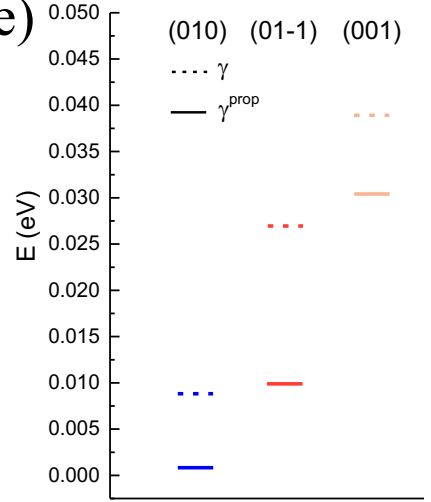

Figure 2. a) SEM micrograph of Se nanowires growing from an amorphous matrix. The sample was soaked in 1-propanol for 12 hours. b) Bright field TEM image and SAED pattern (inset). c) HRTEM image of a single nanowire taken from a fiber cross-section that was soaked in 1-propanol for 5 days. d) Se crystal shape and definition of ah and ch parameters. e) Surface free energies of (010), (01-1) and (001) planes of crystals in contact with 1-propanol molecules or not.

$=0.027$ and $\gamma(001)=0.039 \mathrm{eV} / \AA^{2}$ (Figure 2e). By minimizing the overall surface energy for a given volume, the optimum ch/ah ratio (Figure 2d) was found to be 7.7, which is consistent with the elongated habit of native Se crystals, but is not compatible with the extreme aspect ratio of nanowires.

We then investigated the effect of immersion in 1-propanol, and evaluated the Se/1-propanol interfacial energy by $\gamma_{\mathrm{s}}^{\text {prop }}=\gamma_{s}+\gamma_{\text {prop }}-E / A$, where $\gamma_{\text {prop }}=1.4 \mathrm{meV} / \AA^{2}$ is the (small) surface tension of 1-propanol. We then roughly estimated the work of adhesion by computing the binding energy E of one 1-propanol molecule on a given surface, and its contact area with that surface (see details in SI2). The interfacial 
energies between Se and 1-propanol are $\gamma(010)=0.001, \gamma(01-1)=0.010$ and $\gamma(001)=0.031 \mathrm{eV} / \AA^{2}$ (Figure 2e), resulting in a predicted aspect ratio ch/ah of the crystals of about 24 . While the crude assumptions behind our estimate prevent us from drawing quantitative conclusions, it is clear that using 1-propanol as a solvent modulates the anisotropy of the surface energy, favoring the growth of highly anisotropic crystals, elongated along their trigonal axis. On the basis of ab initio modeling and supported by our experimental observation, we can hence propose the following mechanism for nanowire growth in our system: (i) sonication induces and accelerates the nucleation due to the enhanced mobility of Se atoms; (ii) Se atoms dissolved in the solvent are more energetically favored to attach to crystalline nuclei; (iii) nuclei readily grow into high aspect ratio nanowires with trigonal phase along the [001] direction because the solvent exacerbates the anisotropy of surface free energy of prismatic and basal planes; (iv) nanowires grow at the expense of the amorphous matrix.

Next we turn towards the optical and optoelectronic properties of the nanowire-based devices. First we investigated the optical response of the nanowire network by measuring the optical absorption spectra in the visible and near infrared of meshes grown on glass slides (See SI3). A strong absorption is measured below $630 \mathrm{~nm}$ (Figure S3, SI), corresponding to a weak reflection and hence a strong incoupling of the incoming radiation. This can be attributed to an anti-reflection effect of the porous nanowire mesh that leads to a drastically reduced reflection at the air-semiconductor interface compared to flat Se films. The nanowire mesh also grows only over a thickness of around $500 \mathrm{~nm}$ even for very long immersion time (Figure S2, SI), and yet strongly absorbs incoming light in the visible. Varying the immersion time enables us to tune the depth of the nanowire mesh and hence also its optical and optoelectronic responses, thus affecting the performance of the device. Such a thin photoconductive domain is promising as it exhibits low dark current and associated noise, while can provide excellent responsivity. Indeed, a noise equivalent power is measured to be $3.1 \mathrm{nWHz}^{-1 / 2}$ for $\mathrm{V}=10 \mathrm{~V}, \lambda=532 \mathrm{~nm}$ 
and a bandwidth of $1 \mathrm{~Hz}$ for nanowires grown on fibers in Figure 1 (see SI4 for details), which is on par with in-fiber devices demonstrated so far.

Nanowire interconnections are known to impede charge collection. ${ }^{[30]}$ A simple way to improve device performance would be to reduce the electrode spacing down to the typical size of the nanowires, so that generated charges experience less interconnections on their path and could potentially be transported along a single nanowire. We hence fabricated a second multi-material fiber (the hybrid fiber) in which two pieces of the precursor fiber were re-drawn. In the same token, we demonstrate the strong potential of our method by integrating a step-index optical waveguide in the fiber core, to fabricate an all-fiber-integrated fluorescent imaging system we will discuss below. Figure 3a shows a schematic of the hybrid preform. The optical waveguide structure consists of poly(methyl methacrylate) (PMMA) and polycarbonate (PC), which have a slight index contrast. The Se-based precursor fibers were placed between conducting $\mathrm{CPC}$ rings, each in contact with a metallic electrode (eutectic alloy $\mathrm{Sn}_{91} \mathrm{Zn}_{9}$ ) that ensures a good axial conductivity and straightforward electrical contacting (see details in SI5). As shown in Figure $3 b$, the fiber preserves the original cross-section uniformly along tens of meters. The same solution-based approach employed previously was applied to the amorphous Se domains (Figure S5, SI) of the hybrid fiber tip. In Figure $3 b$ (right), it can be observed that a highly dense array of Se nanowires grew in-between and in contact with the CPC electrodes that have now a spacing reduced to around 15 $\mu \mathrm{m}$. In Figure $3 \mathrm{c}$, we compare the photoresponsivity and the ratio $\mathrm{I}_{\mathrm{ph}} / \mathrm{I}_{\mathrm{dark}}$ measured at fixed voltage $(\mathrm{V}$ $=10 \mathrm{~V}$ ) and under monochromatic illumination $(\lambda=532 \mathrm{~nm}$ ) as a function of power (see SI4), for the precursor and hybrid fibers before and after nanowire growth. As expected, the amorphous devices exhibit a much lower response, the ones in the hybrid fiber benefiting from the higher electric field due to the reduced electrode distance. As we anticipated, the performance of the hybrid fiber is drastically increased. Specifically, the responsivity reaches $0.06 \mathrm{~A} / \mathrm{W}$ 
(a)

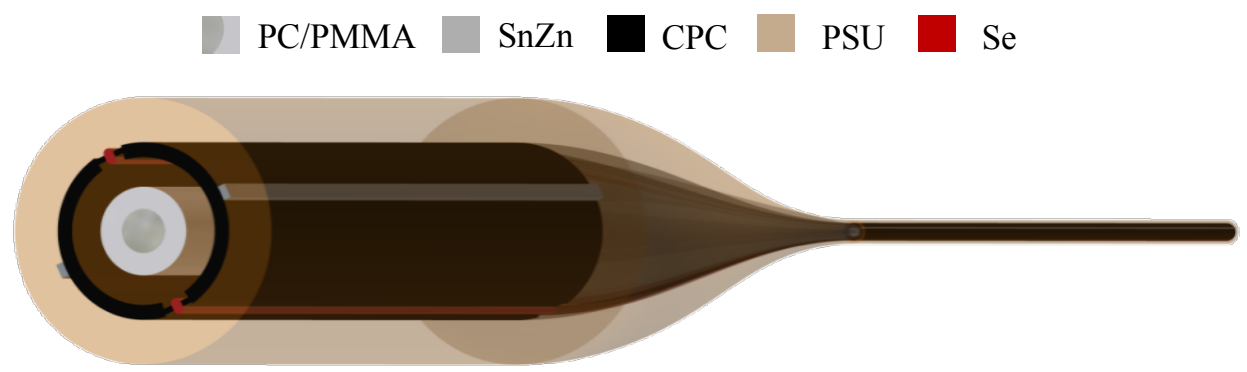

(b)
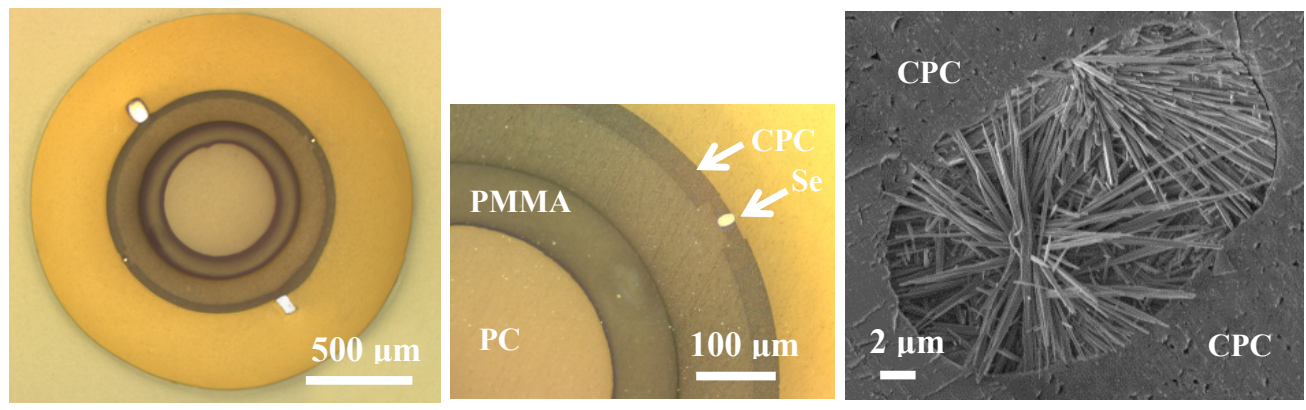

(c)
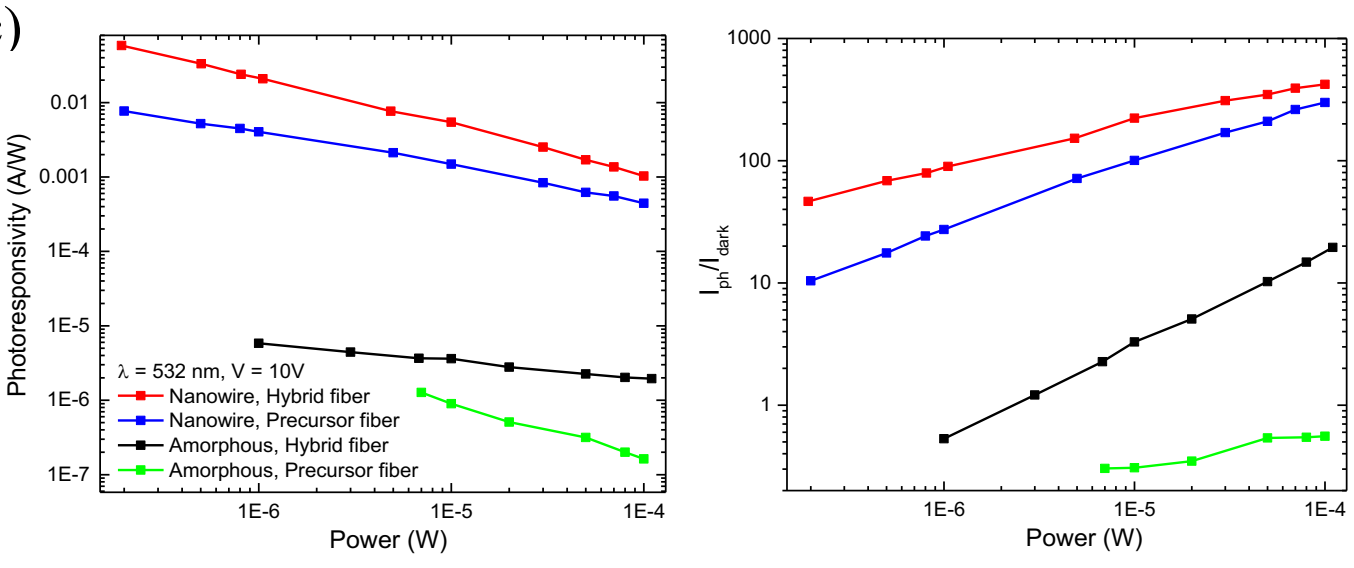

Figure 3. a) Schematic of the thermal drawing of a hybrid fiber. b) Left: optical photograph of the crosssection of the fiber; Middle: high magnification photograph of the redrawn Se region; Right: SEM micrograph of Se nanowires grown between the CPC contacts. c) Left: comparison of photoresponsivity of amorphous-based and nanowire-based precursor and hybrid fibers. Right: ratio $\mathrm{I}_{\mathrm{ph}} / \mathrm{I}_{\mathrm{dark}}$ versus power for the same fibers.

at a power of $200 \mathrm{nW}$ which is almost an order of magnitude better compared to the previous fiber. It reaches $0.1 \mathrm{~A} / \mathrm{W}$ at lower powers, which is comparable to or higher than that of previously reported in- 
fiber photodetectors. ${ }^{[5-7,14,17]}$ The $\mathrm{I}_{\mathrm{ph}} / \mathrm{I}_{\mathrm{dark}}$ ratio also significantly increases, and we measured a noiseequivalent power of $76 \mathrm{pWHz}^{-1 / 2}$ for $\mathrm{V}=10 \mathrm{~V}, \lambda=532 \mathrm{~nm}$ and a bandwidth of $1 \mathrm{~Hz}$, an order of magnitude better than for the best reported in-fiber photoconductors. ${ }^{[5-7,17]}$ This good optical response comes also with an excellent response speed, with a measured rise time of $5.5 \mu$ s (Figure S6, SI5), on par with the best reported value for thermally drawn fibers ${ }^{[17]}$ and several orders of magnitude faster than for other nanoscale devices (Table S2, SI6). Combining excellent sensitivity and fast response speed is unprecedented for thermally drawn in-fiber devices, and makes them comparable to some commercial planar photodetectors, opening great opportunities for optoelectronic fibers.

We now turn to the hybrid fiber described in Figure 3a, a good example of the intriguing functionalities that we can achieve with high performance fiber-integrated optoelectronic devices. The unique combination of optical guidance and high photosensitivity capable of nW-level optical power detection allows us to use the hybrid fibers for both guiding the excitation and detecting a weak fluorescence signal directly at the fiber tip, as shown schematically in Figure 4a (see details in SI7). This contrasts with conventional systems that require the signal to be coupled into an optical fiber and guided to a photodetector at a distal end. To demonstrate this functionality, we fabricated an array of microfluidic channels that represent the letters "EPFL". We filled these channels with a Rhodamine B-based solution (Figure 4b, top) and scanned our hybrid fiber above it. The fiber was guiding an excitation light of 500$\mathrm{nm}$ wavelength, and the current generated in the nanowire-based devices was simultaneously recorded (see the Experimental section). The high sensitivity of the fiber-integrated device enabled to image the EPFL logo accurately (Figure 4b), paving the way towards increasingly complex functional fibers and optical probes.

Finally, an important aspect of thermally drawn fibers is the scalability of the process and their ability to cover and functionalize large surfaces. The fabrication scheme we have demonstrated so far turns out to also be compatible with the integration of nanowires along the fiber length. With a judicious choice 
of material and preform architecture, ${ }^{[31]}$ a Se domain sandwiched between two electrodes can be exposed along the fiber axis. Figure 4c shows the schematic of such a configuration (see details in SI7). Exposing such fibers to the same procedure described above, a nanowire mesh could be grown along extended fiber length, in contact with the CPC electrodes as shown on the SEM micrographs in Figure 4d. . The photo-response of these nanowires was measured to be on par with the wires grown on the fiber tip (see SI7), opening great opportunities for large area, flexible and textile integrated optoelectronic devices.

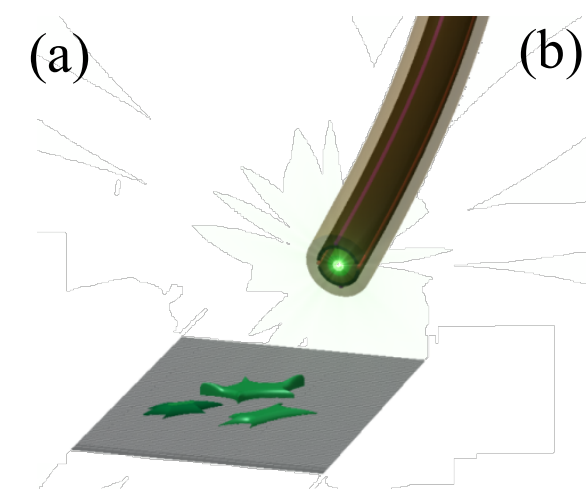

(b)
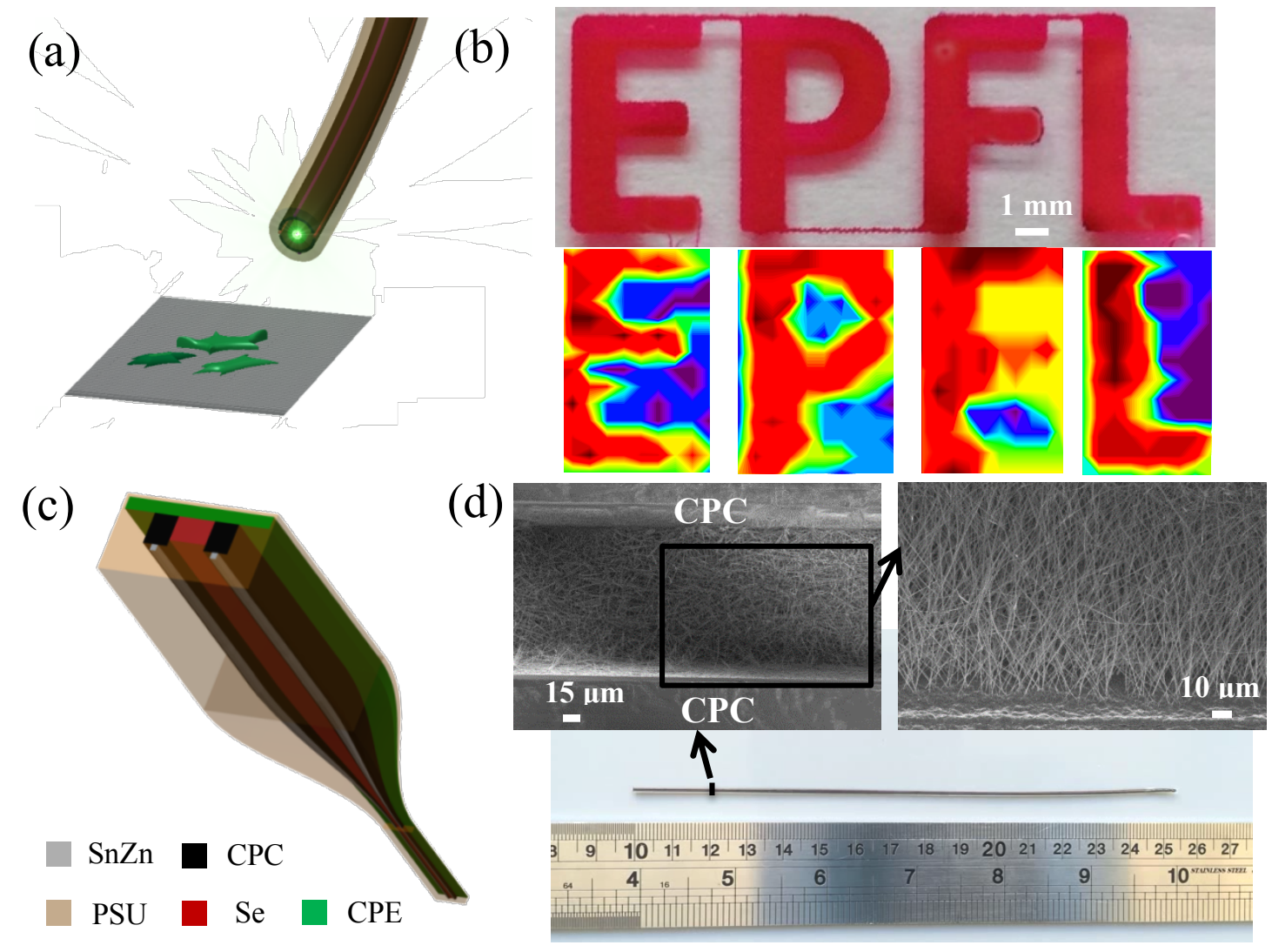

Figure 4. a) Schematic of the fluorescence imaging system. b) Top: photograph of the "EPFL" logo filled with dye-Rhodamine B dissolved in ethanol (captured with a Vidicon camera); bottom: fluorescent image obtained with the hybrid fiber device. c) Schematic of the thermal drawing of a fiber for growing Se nanowires along the fiber length. d) Photograph of a $15 \mathrm{~cm}$-fiber and SEM micrographs showing the Se nanowire mesh and its interface with a CPC electrode. 
In summary, we have developed a simple approach for the fabrication of nanowire-based optoelectronic devices within multi-material fibers. The nanowire growth mechanism was elucidated via electron microscopy as well as first-principles calculations. We could demonstrate fiber-integrated devices with unprecedented optoelectronic characteristics, with nanowires grown on their cross-section but also along their extended length. This nanowire platform brings a novel way to integrate functional domains within flexible fibers. Other materials and functionalities could be investigated such as the integration of junctions for photodiodes or photovoltaic configurations, as well as piezoelectric or thermoelectric materials. This opens unforeseen opportunities for advanced optical fiber probes and imaging systems in bioengineering and health care, in remote and distributed sensing, energy harvesting, and advanced textiles.

\section{Experimental Section}

SEM and TEM samples making and characterization: All the SEM samples were coated with a $10 \mathrm{~nm}$ carbon film. The SEM images were taken with a Zeiss Merlin field emission SEM (Zeiss, Göttingen, Germany) equipped with a GEMINI II column operating at $2.0 \mathrm{eV}$ with a probe current of $150 \mathrm{pA}$. The TEM samples were prepared by embedding them in epoxy resin followed by sectioning thin slices $(60 \mathrm{~nm})$ using ultramicrotomy (diamond blade) which were transferred on a carbon/Cu grid support (300 Mesh). The TEM images and selected area electron diffraction (SAED) patterns were taken using a Talos F200X operating at $200 \mathrm{kV}$.

Photoelectric measurements: The electrical- and photoresponse were measured using a Keithley 6517B. A SuperK Extreme (S/N: 14390105) from NKT Photonics was used as light source. The laser beam used to characterize the optoelectronic properties of the devices was incoming perpendicularly to the fiber cross-section (hence parallel to the fiber axis), for the nanowire grown on the fiber cross-section. For the nanowire grown on the fiber side, side illumination was used. The optical laser power was adjusted using an afocal lens system and was measured using a laser power meter (Thorlabs PM 100D). The time 
resolved responses of the devices were measured using a pulsed LED with a frequency of $100 \mathrm{~Hz}$, and an oscilloscope was used to monitor the variation of current with time.

Fluorescence imaging: The "EPFL" logo microfluidic channel was fabricated in poly(dimethyl siloxane) (PDMS) using standard soft lithography. A hybrid fiber was used to guide the incident light generated from the SuperK Extreme (S/N: 14390105). The fiber was mounted with a $30^{\circ}$ angle with respect to the horizontal surface of the PDMS channel to avoid unwanted reflection. The incident laser was at 500-nm, and the emission peak from the dye was at 570-nm.

\section{Supporting Information}

Supporting Information is available from the Wiley Online Library or from the author.

\section{Acknowledgements}

We also would like to acknowledge the Swiss National Science foundation (grant 200021_146871), and the European Research Council (ERC Starting Grant 679211 "FLOWTONICS") for their funding support.

\section{References}

[1] M. Alexander Schmidt, A. Argyros, F. Sorin, Adv. Opt. Mater. 2016, 4, 13.

[2] R. Chen, A. Canales, P. Anikeeva, Nat. Rev. Mater. 2017, 2, 16093.

[3] M. Bayindir, A. F. Abouraddy, F. Sorin, J. D. Joannopoulos, Y. Fink, Opt. Photon. News 2004, 15, 24.

[4] A. M. Stolyarov, A. Gumennik, W. McDaniel, O. Shapira, B. Schell, F. Sorin, K. Kuriki, G. Benoit, A. Rose, J. D. Joannopoulos, Y. Fink, Opt. Express 2012, 20, 12407.

[5] A. Gumennik, A. M. Stolyarov, B. R. Schell, C. Hou, G. Lestoquoy, F. Sorin, W. McDaniel, A. Rose, J. D. Joannopoulos, Y. Fink, Adv. Mater. 2012, 24, 6005. 
[6] F. Sorin, A. F. Abouraddy, N. Orf, O. Shapira, J. Viens, J. Arnold, J. D. Joannopoulos, Y. Fink, Adv. Mater. 2007, 19, 3872.

[7] F. Sorin, O. Shapira, A. F. Abouraddy, M. Spencer, N. D. Orf, J. D. Joannopoulos, Y. Fink, Nano Lett. 2009, 9, 2630.

[8] G. Kostovski, P. R. Stoddart, A. Mitchell, Adv. Mater. 2014, 26, 3798.

[9] W. Zeng, L. Shu, Q. Li, S. Chen, F. Wang, X. M. Tao, Adv. Mater. 2014, 26, 5310.

[10] N. D. Orf, O. Shapira, F. Sorin, S. Danto, M. A. Baldo, J. D. Joannopoulos, Y. Fink, Proc. Natl. Acad. Sci. 2011, 108, 4743.

[11] Z. Zhang, Q. Zhang, K. Guo, Y. Li, X. Li, L. Wang, Y. Luo, H. Li, Y. Zhang, G. Guan, B. Wei, X. Zhu, H. Peng, J. Mater. Chem. C 2015, 3, 5621.

[12] W. Weng, P. Chen, S. He, X. Sun, H. Peng, Angew. Chemie. Int. Ed. 2016, 55, 6140.

[13] Z. Zhang, K. Guo, Y. Li, X. Li, G. Guan, H. Li, Y. Luo, F. Zhao, Q. Zhang, B. Wei, Q. Pei, H. Peng, Nat. Photonics 2015, 9, 233.

[14] R. He, P. J. A. Sazio, A. C. Peacock, N. Healy, J. R. Sparks, M. Krishnamurthi, V. Gopalan, J. V. Badding, Nat. Photonics 2012, 6, 174.

[15] N. Healy, S. Mailis, N. M. Bulgakova, P. J. A. Sazio, T. D. Day, J. R. Sparks, H. Y. Cheng, J. V Badding, A. C. Peacock, Nat. Mater. 2014, 13, 1.

[16] A. C. Peacock, U. J. Gibson, J. Ballato, Adv. Phys. X 2016, 1, 114.

[17] L. Wei, C. Hou, E. Levy, G. Lestoquoy, A. Gumennik, A. F. Abouraddy, J. D. Joannopoulos, Y. Fink, Adv. Mater. 2017, 29, 1603033.

[18] D. A. Coucheron, M. Fokine, N. Patil, D. W. Breiby, O. T. Buset, N. Healy, A. C. Peacock, T. Hawkins, M. Jones, J. Ballato, U. J. Gibson, Nat. Commun. 2016, 7, 13265.

[19] N. Healy, M. Fokine, Y. Franz, T. Hawkins, M. Jones, J. Ballato, A. C. Peacock, U. J. Gibson, $A d v$. Opt. Mater. 2016, 4, 1004. 
[20] S. Danto, F. Sorin, N. D. Orf, Z. Wang, S. A. Speakman, J. D. Joannopoulos, Y. Fink, Adv. Mater. 2010, 22, 4162 .

[21] D. J. Lipomi, R. V. Martinez, M. A. Kats, S. H. Kang, P. Kim, J. Aizenberg, F. Capasso, G. M. Whitesides, Nano Lett. 2011, 11, 632.

[22] S. Chaudhary, A. Umar, S. K. Mehta, Prog. Mater. Sci. 2016, 83, 270.

[23] K. S. Suslick, Science 1990, 247, 1439.

[24] B. T. Mayers, K. Liu, D. Sunderland, Y. Xia, Chem. Mater. 2003, 15, 3852.

[25] B. Gates, B. Mayers, B. Cattle, Y. Xia, Adv. Funct. Mater. 2002, 12, 219.

[26] P. Cherin, P. Unger, Inorg. Chem. 1967, 6, 1589.

[27] A. Tkatchenko, R. A. Distasio, R. Car, M. Scheffler, Phys. Rev. Lett. 2012, 108, 1.

[28] A. Ambrosetti, A. M. Reilly, R. A. Distasio, A. Tkatchenko, J. Chem. Phys. 2014, 140, 18 A508. [29] V. Blum, R. Gehrke, F. Hanke, P. Havu, V. Havu, X. Ren, K. Reuter, M. Scheffler, Comput. Phys. Commun. 2009, 180, 2175.

[30] S. Ye, A. R. Rathmell, Z. Chen, I. E. Stewart, B. J. Wiley, Adv. Mater. 2014, 26, 6670.

[31] T. Nguyen-Dang, A. C. de Luca, W. Yan, Y. Qu, A. G. Page, M. Volpi, T. Das Gupta, S. P. Lacour, F. Sorin, Adv. Funct. Mater. 2017, 27, 1605935. 


\section{Table of Contents}

\section{Semiconducting Nanowire-based Optoelectronic Fibers}

Wei Yan, Yunpeng Qu, Tapajyoti Das Gupta, Arouna Darga, Dang Tùng Nguyên, Alexis Gérald Page, Mariana Rossi, Michele Ceriotti, and Fabien Sorin*

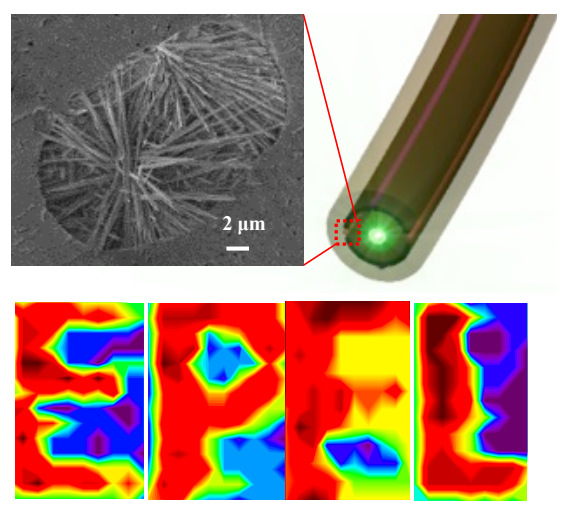

High quality single crystal semiconducting nanowire-based optoelectronic devices, for the first time, are integrated into the tip of polymer optical fiber and along the fiber length. The fiber-integrated devices, fabricated via a combination of simple and scalable approaches, exhibit unprecedented optoelectronic properties. To highlight the opportunities of our approach in advanced fibers and textiles, we demonstrate an all fiber-integrated fluorescence imaging system. 